\title{
Revisiting the Evaluation of Segmentation Results: Introducing Confidence Maps
}

\author{
Christophe Restif \\ Department of Computing, Oxford Brookes University, Oxford OX33 1HX, UK \\ christophe.restif@centraliens.net
}

\begin{abstract}
We introduce a novel framework, called Confidence Maps Estimating True Segmentations (Comets), to store segmentation references for medical images, combine multiple references, and measure the discrepancy between a segmented object and a reference. The core feature is the use of efficiently encoded confidence maps, which reflect the local variations of blur and the presence of nearby objects. Local confidence values are defined from expert user input, and used to define a new discrepancy error measure, aimed to be directly interpreted quantitatively and qualitatively. We illustrate the use of this framework to compare different segmentation methods and tune a method's parameters.
\end{abstract}

\section{Introduction}

Evaluating a segmentation method is important, necessary, and difficult to define. It is important to assess quantitatively a method's results to validate it, for example on clinical data sets. It is necessary to rank segmentation results, when tuning parameters or comparing methods. However, evaluation in itself is difficult to define: it depends on the definition and purpose of the segmentation, and on the types of errors relevant for the task. Additional issues affect medical imaging. Partial volume effect and blur cause ambiguity on the actual location of borders, and expert variability lead to multiple references for the same objects.

Recent publications have defined evaluation criteria. In [1], Zhang describes three types of evaluation. Analysis focuses on the algorithmic part of segmentation; goodness evaluates results using image and object properties, with no external reference; discrepancy compares segmentation results to references (or ground truth). In 2], Udupa et al. emphasise that evaluation depends on the application domain: the application or task considered, the body region imaged, and the imaging protocol. Each application domain has specific properties, such as the type of noise in images, which affect the choice of an evaluation method. Yet, some issues remain open: in particular, how to define a reference and cope with multiple references, and how to measure the agreement between a segmented object and a reference. This article focuses on discrepancy evaluation, and presents our contributions to those open issues, in the context of two-dimensional images containing multiple objects affected by blur or partial volume effect. For illustration we use examples from cytometry, where these effects are common.

N. Ayache, S. Ourselin, A. Maeder (Eds.): MICCAI 2007, Part II, LNCS 4792, pp. 588 595, 2007.

(C) Springer-Verlag Berlin Heidelberg 2007 
References for segmentation are often defined as binary masks, drawn by experts or computed from segmentations. Different experts, or a given expert at different times, may disagree on some parts of objects' boundaries: those effects are termed inter- and intra-user variability. Although keeping the different references of a single object can be useful, in particular to analyse these variabilities [3,4, some applications require a single reference per object, which calls for a way of merging multiple references [5, 6, 4, One strategy is to average the pixel distance between the borders of the multiple references [7]. Another is to optimise the overlaps between the multiple references and the result of the merging. STAPLE [4 measures overlaps with specificities and sensitivities, while VALMET [3] uses more diverse measures. Overlaps areas may depend on object and image sizes though, which might be an issue when objects are significantly smaller than images [8], in particular in many cytometry applications. Also, the results of such methods do not indicate the differences between the input references. A solution [5] is to average the binary values of the masks, resulting in a multi-valued mask with as many possible values as there are input references.

The agreement between a segmented shape and a reference may be measured with a variety of methods. Area-based measures classify pixels in different regions (e.g. true and false positives and negatives) and return various ratios of region areas. Distance-based measures focus on the distance between the reference's and the segmented object's borders, and return various statistics on them (e.g. maximum, average, variance). Although they allow method comparisons, such measures may be difficult to interpret. Area-based measures may depend on objects and background sizes, and treat all pixels with the same importance (whether they are in blurred regions or well inside objects). Distance-based and fuzzy measures [9] have to be compared to some reference value, e.g. the width of blur around objcts, which may vary locally. One approach to evaluation is to use several measures [2,3,10. It produces more data, but leads to further questions on how to combine numbers in different units, or on which measures are more informative. Introducing classifiers to combine measures significantly increases the complexity of evaluation, as it creates dependencies on choices of classifiers, parameters and training sets.

Our main contribution is to replace the binary-mask type of reference with a confidence map, storing more information in the reference at low cost in terms of user input and storage. Under this framework, called Confidence maps estimating true segmentations (Comets), similar techniques for merging references and measuring agreements can easily be defined, as described below, with the aim to be more easily interpreted, and more meaningful for some applications.

\section{Method}

Motivation. We focus our work on two-dimensional medical images, showing any number of objects, and affected by blur or partial volume effect. Examples from cytometry are shown in Fig. 1. On these images, objects of interests (1) are surrounded with blurred regions (2) or nearby objects (3), and with background (4) 

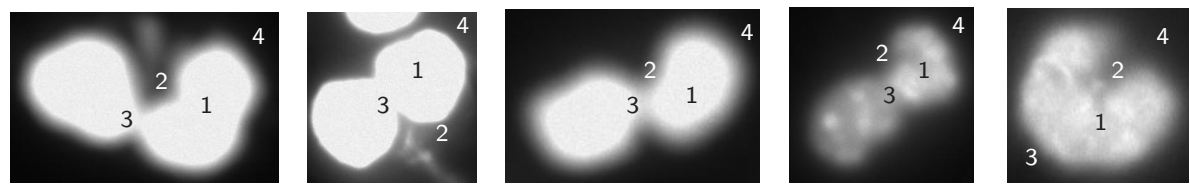

Fig. 1. Local variations of ambiguity around an object. See Section 2 for details.

further away. The location of the object's borders may be unclear in zones (2), but more precise locally (3), due to nearby objects or lower blur. The distance to the object (1) is not enough to discriminate zones (2) and (3), and while the area of zone (2) may be significant compared to zone (1), slight variations of segmentation in zone (2), where a correct reference is difficult to define, may still be acceptable for segmentation applications.

The motivation for using confidence maps as references, is to define an evaluation measure such that the penalty for mis-segmenting the zones of ambiguity (2) is lower than for the other zones, and the penalties for mis-segmenting zones (3) and (4), i.e. under-segmenting the object, can be differentiated from mis-segmenting zone (1), i.e. over-segmenting the object. Specifying where these zones stand is left to the expert user defining the references for the objects. We call the framework Comets, for Confidence maps estimating true segmentations: although the true segmentation of an object may not be known with one-pixel precision, it may be estimated with a confidence map.

Confidence map. For each object, every pixel $P$ is given a real number $\operatorname{Conf}(P)$ reflecting the expert's confidence in where $P$ stands, as follows. Qualitatively,

$$
\begin{cases}\text { if } \operatorname{Conf}(P)=0 & : \mathrm{P} \text { is believed to be on the border of the object } \\ \text { if }-1 \leq \operatorname{Conf}(P) \leq 1 & : \mathrm{P} \text { is believed to be on or close to the border } \\ \text { if } \operatorname{Conf}(P)>1 & : \mathrm{P} \text { is strongly believed to be part of the object } \\ \text { if } \operatorname{Conf}(P)<-1 \quad: \mathrm{P} \text { is strongly believed to be out of the object }\end{cases}
$$

Quantitatively, the higher $|\operatorname{Conf}(P)|$, the more confident the expert is that $\mathrm{P}$ belongs to the object or to the background. The pixels with low confidence, between -1 and 1, form a band around the object's border (see white bands on Fig. 25 and Fig. 4d). The local width of the band reflects the expert's local confidence in the border's actual location: the narrower it is locally, the more confident the expert is that the border drawn corresponds to the actual border. It corresponds to the zone (2) described above. Pixels with confidence above 1 are in zone (1), while pixels with confidence below -1 are in zones (3) and (4).

To avoid storing the whole map on the whole image for each object, we use the following model. Along a line normal to the object's border, the confidences grow linearly, with a linear factor depending both on the location of that line (i.e. where the line intersects the border), and on the direction of the line, either inward or outward. It allows local variations of confidence around the object, and independent confidences for the inside and outside of objects. With this model, each pixel $B$ on the border is assigned two positive parameters, called inner and outer confidence factor. This is enough to compute the confidence of any pixel 

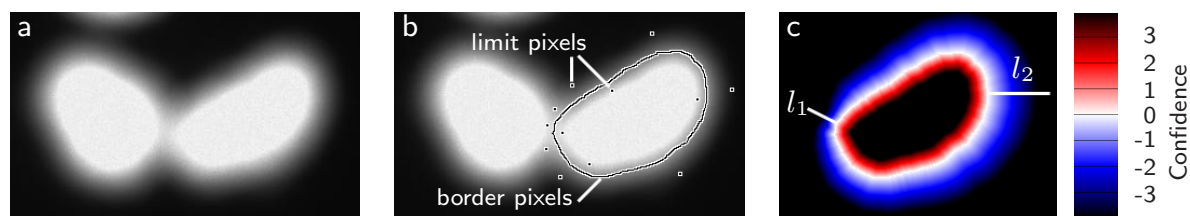

Fig. 2. Construction of a Comets. a: original image; b: user input (border and limit pixels); c: confidence map. Lines $l_{1}$ and $l_{2}$ show regions with different variations of confidence, reflecting the expert's local confidence on the location of the border.

$P$. If $P$ is on the border, $\operatorname{Conf}(P)=0$. Else, let $B$ be the border pixel closest to $P$, at distance $d$ and with confidence factors $I C(B)$ and $O C(B)$. If $P$ is inside the object, $\operatorname{Conf}(P)=\frac{d}{I C(B)}$. If $P$ is outside the object, $\operatorname{Conf}(P)=-\frac{d}{O C(B)}$.

By visualising a confidence map as a height map, the border is the level set 0 , the object stands at positive height (mountain-shaped) and the rest of the image at negative heights. In this height map, the confidence factors stored at each border pixel correspond to the gradients of the slopes, inwards and outwards. These two slopes are independent, and may vary along the border. This is illustrated in Fig. 22: the gradient is sharper along line $l_{1}$ than $l_{2}$.

User input to define Comets. The previous paragraph details how to compute the confidence of any pixel given the border pixels and their confidence factors. However, these factors may not be easy to define manually. We use the following scheme instead. An expert draws a continuous line of pixels (called border pixels) where they think the actual border stands. Then, the expert selects any number of pixels inside the object, called inner limit pixels, which they are certain belong to the object and are as close to the border as possible. These will receive confidence value 1 . Similarly, they select pixels outside the object (referred to as outer limit pixels), as close to the border as possible while confidently belonging to the background. These pixels will have confidence value -1 . An example of user input is shown in Fig. 2 $\mathrm{b}$. Any number of limit pixels can be selected. With this input, the confidence factors are computed as follows. First, for each limit pixel $P$, the closest border pixel $E$, at distance d, is found. If $P$ lies outside the object, $E$ is assigned an outer confidence factor of $d$. If $P$ is inside, $E$ is assigned an inner confidence factor of $d$. The resulting inner and the outer confidence factors are both linearly interpolated along the curvilinear coordinate describing the border. This method is illustrated in Fig. 3. Fig. 3a shows part of the border and two outer limit pixels. Fig. 3b shows the assignment of the outer confidence factor to the two closest border pixels, and the linear interpolation between them. Fig. 3r illustrates how the confidence of a pixel is computed with the confidence factors.

Evaluating segmentation results. We present a single measure to reflect the severity and type of segmentation error. Let $C$ be a Comets reference and $O b j_{C}=\{P: \operatorname{Conf}(P) \geq 0\}$ the set of pixels referenced as the object. Let $S$ be 

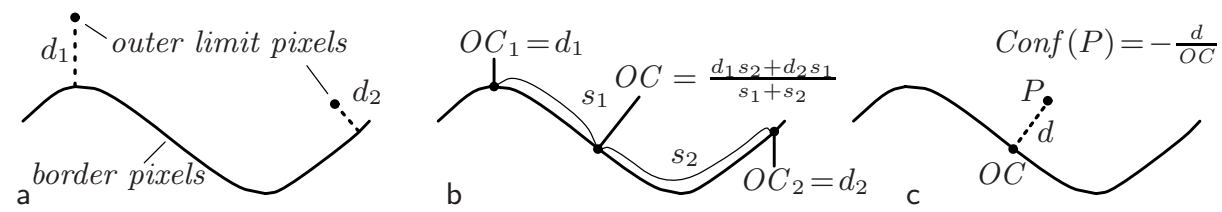

Fig. 3. Linearisation of confidence factors. a: user input; b: Outer Confidence factors assigned to the border pixels closest to outer limit pixels then interpolated linearly along the curvilinear coordinate; c: Confidence of any outside pixel $P$, based on the Outer Confidence factor of the closest border pixel.

the set of pixels segmented as the object, and $M i s=\left(S \cup O b j_{C}\right)-\left(S \cap O b j_{C}\right)$ the set of mis-labeled pixels. The error of $S$ against $C$ can be defined as:

$$
\operatorname{error}(S, C)=\left\{\begin{array}{l}
\infty \text { if } S \cap O b j_{C}=\emptyset \\
\operatorname{Conf}\left(P_{0}\right), P_{0}=\arg \max _{P \in M i s}\{|\operatorname{Conf}(P)|\} \text { else. }
\end{array}\right.
$$

Intuitively, if there is no overlap between the segmented object and the reference, they are not related and the error is $\infty$. Otherwise, mislabeling pixels of low confidence only (near the object boundary) leads to a small error, while mislabeling higher confidence pixels causes a greater error. Moreover, the value of this error can be interpreted in terms of qualitative segmentation error 11, as follows. A shape with error 0 is a perfect match for the reference. An error within -1 and 1 indicates that only pixels of low confidence were mislabeled: the segmentation can be considered correct. An error greater than 1 shows that object pixels were labeled as background, so the shape is over-segmented. An error below -1 is due to background pixels labeled as object: the shape is under-segmented. When a segmented object both over- and under-segment a reference, this single measure keeps the worse error of the two. Should more information be needed, the error measure can be extended, for example by returning a couple of values: the maximum positive and the minimum negative confidence of the mis-labelled pixels. This reflects the severity of the over- and under-segmentation of each object, with the same straightforward interpretation as above.

Combining multiple references. As explained in the introduction, it is sometimes necessary to merge multiple expert references into a single entity before evaluating a segmentation result. Under the Comets framework, references can be weighted with their local confidences, by simply averaging the confidence values of each map. Let $\left\{C_{i}\right\}_{1 \leq i \leq n}$ be the set of $n$ expert segmentations to combine, encoded as Comets. Let $\Omega=\left\{P, \exists i\right.$ : $\left.\operatorname{Conf}_{i}(P) \geq-1\right\}$, where $\operatorname{Conf}_{i}(P)$ is the confidence of pixel $P$ with respect to $C_{i}$. All pixels outside $\Omega$ are confidently considered as background by all the experts, so they are discarded for the rest of the construction. The combined confidence map on the domain $\Omega$ is the average of all the experts' confidence maps: $\forall P \in \Omega, \operatorname{Conf}(P)=\frac{1}{n} \sum_{i=1}^{n} \operatorname{Conf}_{i}(P)$. Let $\{P \in \Omega: \operatorname{Conf}(P)=0\}$ be the resulting border pixels, $\{P \in \Omega: \operatorname{Conf}(P)=-1\}$ the resulting outer limit pixels, and $\{P \in \Omega: \operatorname{Conf}(P)=1\}$ the resulting inner limit pixels. The confidence factors of the resulting border pixels are computed 

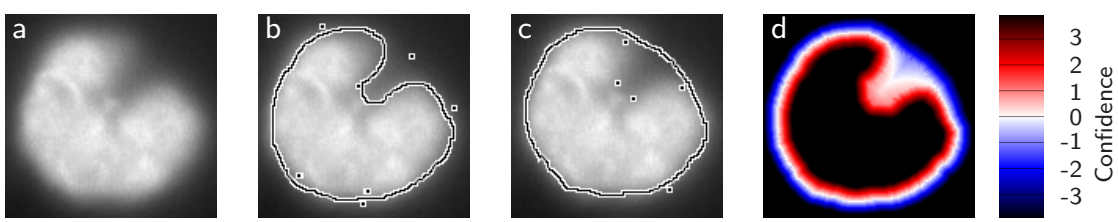

Fig. 4. Combining multiple segmentations. a: original image; b: input from expert 1; c: input from expert 2; d: resulting average Comets.

as detailed above. An example of reference combination is shown in Fig. 4. The zones of disagreement receive a lower confidence, in absolute value, than the zones of agreement, allowing the segmentation errors there to be less penalised.

Comparison and tuning of segmentation methods. Quantitative evaluation allows the comparison of different segmentation methods, and different sets of parameters for each method. To illustrate the comparatively straightforward interpretation of the Comets measure defined in Eq. (11), we use three segmentation methods on the same data set of cell images, based on local thresholding [12, watershed and active contours 13 . The results for the first two are shown in the graph of Fig. 5 a , as the number of objects ( $y$ axis) having an error between -10 and 10 ( $x$ axis). The orange rectangle shows the acceptable error zone between -1 and 1. The watershed, in blue, has a high number of errors between 1 and 3 , reflecting over-segmentation, and on this data set the thresholding technique performs better than the watershed. In Fig. 5b, four sets of parameters for the active contours are compared. Set 1, in blue, has the higher proportion of correctly segmented shapes (in the orange zone), and is better than the others on the data set used. Although these results can be analyzed in more detailed, it is beyond the scope of this article.

\section{Discussion}

As detailed in 2], an evaluation method should be designed according to a segmentation method, which makes it difficult to compare evaluation methods in general. In this section we examine their differing principles. Tools such as STAPLE 4 and VALMET 3] are designed for 3D data sets, where objects occupy the major part of the image. This assumption does not always hold for $2 \mathrm{D}$ images, especially from microscopy. Also, blur and multiple nearby objects can cause local variations of ambiguity. Two important consequences for evaluation are that some pixels may be less important to segment correctly than others, and those are not merely characterised by their distances to the object's border, as illustrated in Fig. 1] The former observation lowers the relevance of area-based evaluation measures, and the latter, that of distance-based measures. Our approach is to encode these variations within the references, to simplify the choice and interpretation of evaluation measures. To the best of our knowledge, the closest work is from Jin et al. [5] who define multi-valued references as the average of binary masks, and use these values to weight the measure. However, the 

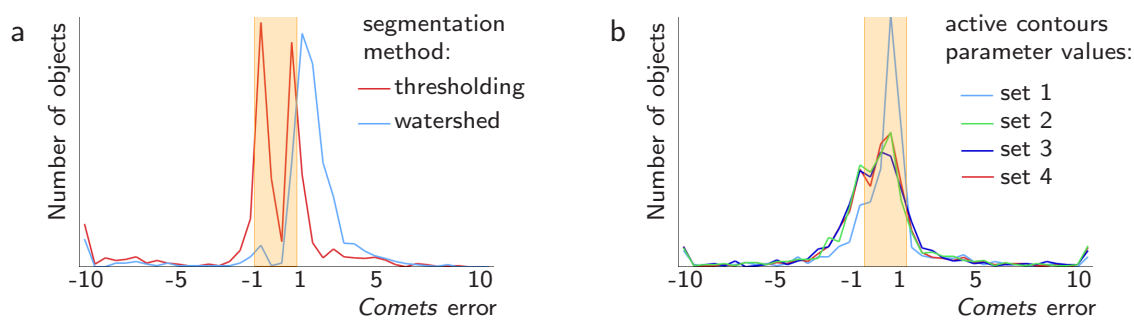

Fig. 5. Use of the single Comets error measure to (a) compare segmentation methods and (b) tune one method's parameters. See Section 2 for details.

values are discrete, and only the result of reference merging. Also, non-binary values only occur where different experts disagree: this method is not designed to encode blur, only user variability, and requires several references for each object. Our approach is more general from the beginning: the confidence maps use a continuous range of numbers, and so for every reference.

We presented a single error measure to reflect the severity and type of segmentation error. With the type of images we use here, such an interpretation is difficult to obtain from single area- or distance-based measures. Although combining multiple measures may improve the interpretation, it also increases the complexity of evaluation. Another approach to reflect the types and severities of errors from evaluation results is presented in [11,14]. Each segmented object is classified (as over-, under-segmented, noise, etc.) by comparing its relative overlap with a reference, with a threshold value between $50 \%$ and $100 \%$. Plotting the number of objects in one category as a function of the threshold chosen, produces a curve reflecting the quality of the segmentation on the whole data set. For comparison or tuning of segmentation methods, the areas below several sets of $2 \mathrm{D}$ curves have to be compared. Although this method presents more data, it may not be straightforward to use because of its complexity. With graphs as in Fig. 5, the performance of different methods may be easier to compare.

\section{Conclusion}

We have presented a novel framework, Comets, that allows:

- the encoding of segmentation references with local confidence factors, storing more expert knowledge than a binary mask at low cost;

- the combination of multiple references, with confidence-based local weights; - a single discrepancy measure with a straightforward interpretation, both qualitative (e.g. under-, over-segmented) and quantitative (severity).

The framework is based on local confidence factors, which are derived from an expert user's input. The extra input needed, namely the selection of limit pixels, is less demanding that landmark selection, and could be readily added to existing systems. We have illustrated how this framework can be used to compare qualitatively and quantitatively different segmentation methods applied to 
the same set of images, or to tune one method's parameters. In case more information is needed from the evaluation results, more complex measures can easily be defined, based on the same intuitive interpretation of confidence.

Future work include a quantitative comparison with other evaluation measures, which amounts to evaluating different evaluation methods and is beyond the scope of this article. We also consider using non-linear functions of $d$ to define the confidence $\operatorname{Conf}(P)$, in particular using logarithm odds maps [15].

\section{References}

1. Zhang, Y.J.: A review of recent evaluation methods for image segmentation. In: Intl. Symposium on Signal Processing and Its Applications, Malaysia, pp. 148-151 (2001)

2. Udupa, J., LaBlanc, V., Schmidt, H., Imielinska, C., Saha, P., Grevera, G., Zhuge, Y., Currie, L., Molholt, P., Jin, Y.: Methodology for evaluating image-segmentation algorithms. In: SPIE Medical Imaging, USA, pp. 266-277 (2002)

3. Gerig, G., Jomier, M., Chakos, M.: Valmet: A new validation tool for assessing and improving 3D object segmentation. In: Niessen, W.J., Viergever, M.A. (eds.) MICCAI 2001. LNCS, vol. 2208, Springer, Heidelberg (2001)

4. Warfield, S.K., Zou, K.H., Wells, W.M.: STAPLE: An algorithm for the validation of image segmentation. IEEE Trans. on Medical Imaging 23(7), 903-921 (2004)

5. Jin, Y., Imielinska, C., Laine, A.F., Udupa, J.K., Shen, W., Heymsfield, S.B.: Segmentation and evaluation of adipose tissue from whole body MRI scans. In: Ellis, R.E., Peters, T.M. (eds.) MICCAI 2003. LNCS, vol. 2878, pp. 635-642. Springer, Heidelberg (2003)

6. Rohlfing, T., Maurer, Jr., C.R.: Shape-based averaging for combination of multiple segmentations. In: MICCAI, vol. 2, pp. 838-845. Palm Springs, USA (2005)

7. Tsai, A., Yezzi, Jr., A., Wells, W., Tempany, C., Tucker, D., Fan, A., Eric Grimson, W., Willsky, A.: A shape-based approach to the segmentation of medical imagery using level sets. IEEE Trans on Medical Imaging 22(2), 137-154 (2003)

8. Davis, J., Goarich, M.: The relationship between precision-recall and ROC curves. In: Intl. Conference on Machine Learning, Pittsburgh, USA, pp. 233-240 (2006)

9. Crum, W., Camara, O., Hill, D.: Generalized overlap measures for evaluation and validation in medical image analysis. IEEE Tr. Med. Img. 25(11), 1451-1461 (2006)

10. Klingensmith, J., Shekhar, R., Vince, D.: Evaluation of 3D segmentation algorithms for the identification of luminal and medial-adventitial borders in intravascular ultrasound images. IEEE Trans. on Medical Imaging 19(10), 996-1011 (2000)

11. Hoover, A., Jean-Baptiste, G., Jiang, X., Flynn, P.J., Bunke, H., Goldgof, D.B., Bowyer, K., Eggert, D.W., Fitzgibbon, A., Fisher, R.B.: An experimental comparison of range image segmentation algorithms. IEEE PAMI 18(7), 673-689 (1996)

12. Restif, C.: Towards safer, faster prenatal genetic tests: Novel unsupervised, automatic and robust methods of segmentation of nuclei and probes. In: Leonardis, A., Bischof, H., Pinz, A. (eds.) ECCV 2006. LNCS, vol. 3951, pp. 437-450. Springer, Heidelberg (2006)

13. Restif, C., Clocksin, W.: Comparison of segmentation methods for cytometric assay. In: MIUA, London, UK, pp. 153-156 (2004)

14. Min, J., Powell, M., Bowyer, K.: Automated performance evaluation of range image segmentation algorithms. IEEE Trans on Systems, Man, and Cybernetics - Part B: Cybernetics 34(1), 263-271 (2004)

15. Pohl, K., Fisher, J., Shenton, M., McCarley, R., Grimson, E., Kikinis, R., Wells, W.: Logarithms odds maps for shape representation. In: Larsen, R., Nielsen, M., Sporring, J. (eds.) MICCAI 2006. LNCS, vol. 4190, pp. 955-963. Springer, Heidelberg (2006) 